\title{
Massive Ovarian Enlargement with Multiple Cysts Mimicking Ovarian Neoplasm and Primary Hypothyroidism
}

\author{
REHANA PERVEEN ${ }^{1}$, MIRZA MD. ASADUZZAMAN²
}

\begin{abstract}
Background: Cystic enlargement of ovaries may develop due a large number of causes and may need surgical intervention. Moreover, some ovarian cysts arise due to endocrine disorders \& do not require surgery. We report an unusual case of massive enlargement of ovaries with multiple cysts in a girl with severe hypothyroidism. Morphologic features of cysts on ultrasonography and clinical features of hypothyroidism with elevated TSH level facilitates diagnosis of multiple ovarian cysts and hypothyroidism.
\end{abstract}

Case presentation: A 23-year-old unmarried woman presented with abdominal enlargement, pain and moderate anemia. Abdominal ultra sonogram revealed huge enlargement of the ovaries with multiple large cysts. She was admitted in a hospital, treated with blood transfusion and laparotomy was decided. She was referred to us in a private clinic. On further evaluation , she had features of hypothyroidism with high TSH level and abdomino-pelvic ultrasound revealed enlarged ovaries with multiple large thin-walled cysts and mild ascitis. Conservative management with thyroxin replacement therapy was given and after 3 weeks of thyroxine $\left(T_{4}\right)$ therapy, follow-up abdominal ultrasound showed significant reduction in ovarian size. After 4 months of thyroxine therapy, ovarian size normalized, cysts disappeared completely and there was significant improvement of patient's condition and she became euthyroid.

Conclusion: This case report highlights the rare association between hypothyroidism and multicystic ovarian enlargement. It is necessary to keep in mind the possibility of hypothyroidism in the differential diagnosis of adult patients with ovarian enlargement and multiple cyst formation in order to prevent unnecessary ovarian surgery.

Introduction:

Cystic enlargement of ovaries occur due to a large number of gynecological causes and are often treated by surgery. However, some ovarian cysts develop as a result of endocrine disorders and do not require surgery. Primary hypothyroidism is a common endocrine abnormality resulting from thyroid hormone deficiency that may lead to multiple system impairment. Spontaneous ovarian hyperstimulation might occur following high levels of human chorionic gonadotropin (HCG) in normal pregnancy, high level of thyroid stimulating hormone (TSH) in hypothyroidism and in FSH receptor mutation. Failure to recognize hypothyroidism as an etiology of ovarian cysts could lead to unnecessary oophorectomy.

\section{Case Report:}

A 23-year-old unmarried woman presented with large multi-cystic ovarian enlargement and moderate anemia in a private clinic. She was transfused with blood and advised for laparotomy for ovarian tumor. She was then referred to us. On query she complained of easy fatigability, swelling in hands \& feet with facial puffiness, cold intolerance, weight gain for last one year \& abdominal enlargement, pain \& shortness of breath for last 2 months. There was also complaints of irregular menstrual cycle with excessive flow. On physical examination, she was found to have facial puffiness, swelling in hands \& feet. Her weight was $65 \mathrm{~kg}$ \& deep tendon reflexes were prolonged. Abdominal examination revealed a large cystic lump in lower abdomen extending to umbilical region. A clinical diagnosis of hypothyroidism and cystic

1. Professor, Department of Gynecological Oncology, National Institute of Cancer Research and Hospital, Mohakhali, Dhaka (NICRH).

2. Junior Consultant, Department of Gynecological Oncology, NICRH.

Address of Correspondence: Professor, Department of Gynecological Oncology, National Institute of Cancer Research and Hospital, Mohakhali, Dhaka (NICRH).Email:dr.rehanaperveen@gmail.com 

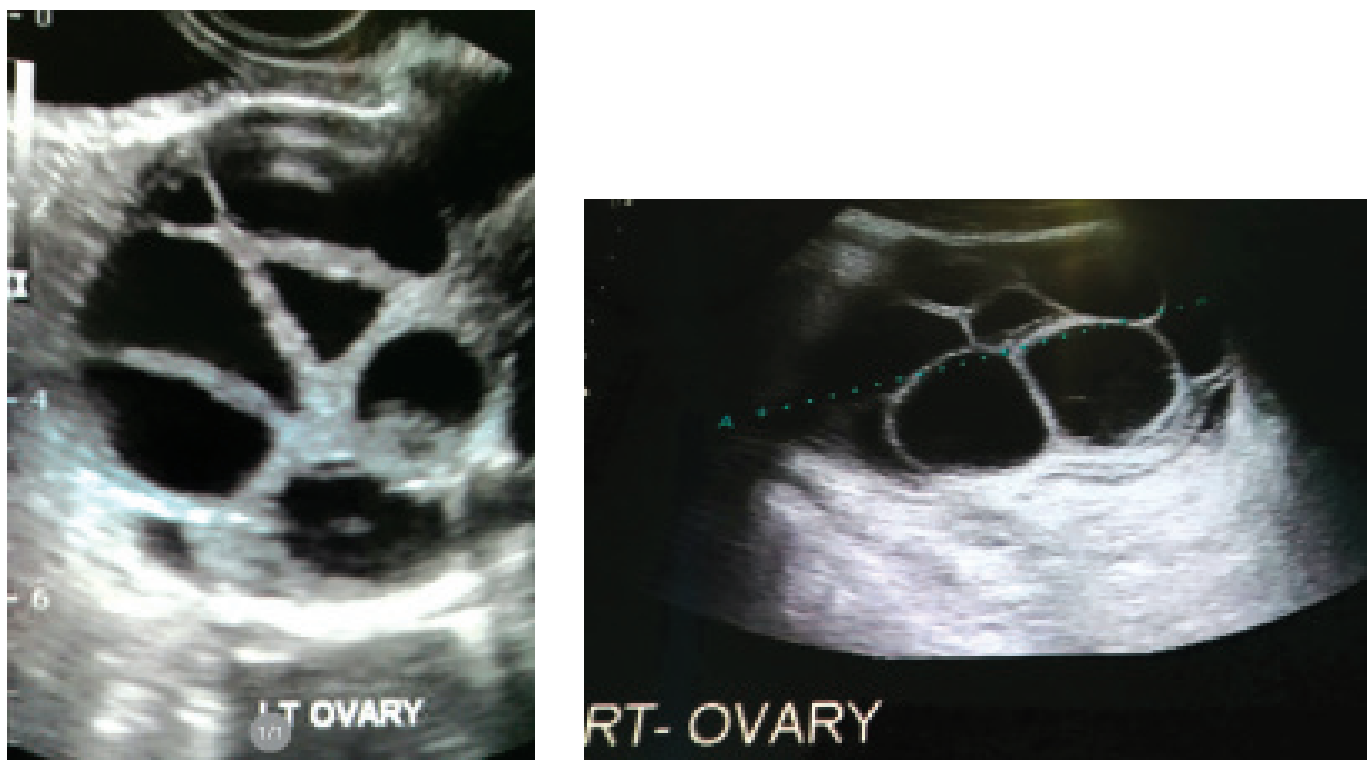

Fig.-1: Sonographic image of right and left ovary before medical treatment.

enlargement of ovaries was made and the patient was investigated further.

Ultrasound scan of abdomen and pelvis revealed bilateral huge enlargement of ovaries : right ovary, $14.5 \times 7.5 \times 6.4 \mathrm{~cm}$, left ovary, $12 \times 6.5 \times 5.9 \mathrm{~cm}$ with multiple large thin-walled cysts (Fig 1,2)and mild ascitis. CA-125 level was 264U/l. Thyroid function test revealed thyroid stimulating hormone (TSH) level above $100.0 \mathrm{mlU} / \mathrm{ml}$ (normal range: $0.34-4.25$ ) and free thyroxine $\left(\mathrm{T}_{4}\right)$ level $0.18 \mathrm{ng} / \mathrm{l}$ (normal range: 0.8 $-1.8 \mathrm{ng} / \mathrm{l})$. No abnormality was detected on thyroid scanning.. Other hematological and biochemical profile of the patient was within normal range. The patient was diagnosed as a case of primary hypothyroidism associated with enlarged ovaries with multiple enlarged ovarian follicular cysts. Conservative treatment with Thyroid replacement therapy was decided \& thyroxine was given with a dose of $1251 / 4 \mathrm{~g} /$ day. The patient's symptoms improved promptly. After 3 weeks of thyroxin treatment there was significant decrease in size of ovaries and cysts. Ultrasound scan of abdomen and pelvis showed significant regression in cysts and ovarian size. She was followed by serial abdominal ultrasound that showed normal ovary size with complete resolution of cysts 4 months after levothyroxine replacement

She became biochemically euthyroid and CA 125 level revert to normal. Her menstrual cycle returned to normal with normal menstrual flow and her body weight decreased to $53 \mathrm{~kg}$. The patient responded well to conservative management without surgical intervention.

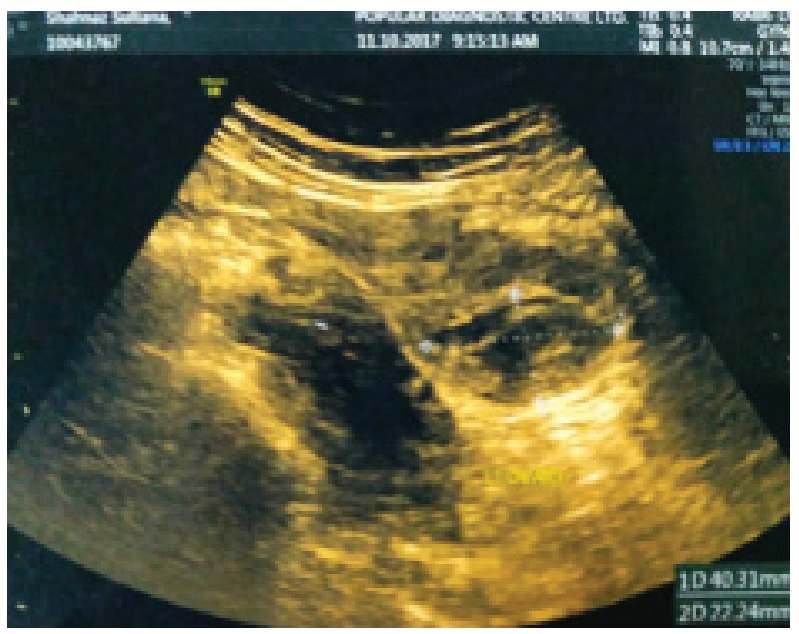

Fig.-2: Sonographic image of regression of ovarian cyst after 4 months of medical treatment.

\section{Discussion:}

Ovarian composition and function is complex and the function is regulated by the integrity of hypothalamo-pituitary-ovarian axis. Etiology of ovarian cyst formation varies greatly including functional cysts either gestational or non-gestational, benign or malignant cysts, cysts of endometriosis or inflammation etc. Some cysts result from endocrine dysfunction and may resolve without surgery after endocrine correction. Ovarian hyperstimulation syndrome (OHSS) is usually iatrogenic and is a 
potentially life- threatening complication of ovulation induction. In some rare cases, spontaneous OHSS related with pregnancy has been described as depending on activating mutations of the $\mathrm{FSH}$ receptors (FSHR) gene, causing ovarian hyperresponsiveness to circulating FSH or even crossresponsiveness of FSHR to hormones having a structure similar to FSH (follicle stimulating hormone), HCG (human chorionic gonadotropin), or TSH ${ }^{1}$. Without considering these endocrine disorders as a possible etiology, a diagnosis of ovarian neoplasm is suspected, leading to unnecessary ovarian resection. Hypothyroidism is one of the important endocrine disorders associated with ovarian hyperstimulation. There have been spontaneous OHSS cases reported in pregnant women with hypothyroidism 2,3,4. Van Wyk \& Grumbach first described the combination of multicystic ovaries, juvenile hypothyroidism and precocious puberty in 1960 , sporadic cases of this syndrome have been reported in pre-pubertal or adolescent girls 5,6,7. However, very few cases have been reported in adults aged 19-26 years ${ }^{8,9}$. We report spontaneous ovarian hyperstimulation in an adult unmarried 23 years old girl with undiagnosed severe primary hypothyroidism.

In premenopausal women, simple ovarian cysts are usually functional and often resolve within 1-2 months without any treatment but large multiloculated or symptomatic cysts usually require treatment. Both ovarian enlargement and large multiloculated cysts are associated with hypothyroidism. Normalization of ovarian size and complete regression of the cysts, together with improvement in serum hormone level, has been shown to occur after the achievement of euthyroidism ${ }^{8}$. Our patient was found to have huge enlargement of ovaries with large multicystic ovarian cysts \& severe hypothyroidism \& this finding is similar to that as described by Jing Shu et al in their case report ${ }^{9}$. Complete regression of the cysts with normalization of ovarian size was achieved after thyroid hormone replacement therapy in our case as described by others, supports a causal relationship between hypothyroidism and ovarian hyperstimulation ${ }^{10}$.

The association of multisystic ovarian disease with hypothyroidism has been described in the literature 9,10,11. Different mechanisms have been postulated, which include altered oestrogen metabolism, hypothalamic-pituitary dysfunction but exact mechanism is not yet established. Anasti et al described in his study that ovarian enlargement in severe hypothyroidism is probably due to the stimulation of FSHRs by unusually high TSH levels proven to have a weak FSH-like activity. It has been shown that TSH could interact directly with the FSHRs to elicit gonadal stimulation, because TSH has a small FSH-and luteinizing hormone-like effect ${ }^{12}$.

Aghajanova et al. described the presence of TSH receptors (TSHR) and thyroid hormone receptors (TRa1, TRa2, TRb1) in human ovarian surface epithelium and in the oocytes of primordial, primary and secondary follicles, which supports the view that TSHR \& TRs may participate in the regulation of ovarian function ${ }^{13}$

Taher BM et al reported in their case, supplementation with thyroid hormone led to complete regression of the multicystic ovarian enlargement, even in patients with high CA 125 levels ${ }^{10}$. Similarly, in our case with thyroid hormone replacement therapy there was complete regression of ovarian cysts and CA 125 level revert to normal.

It is uncertain why hypothyroidism is so common while concomitant ovarian hyperstimulation appears to be so rare. All reported cases are associated with severe hypothyroidism with TSH more than $100 \mathrm{mU} /{ }^{14,15}$. TSH level was also more than $100 \mathrm{mU} / \mathrm{l}$ in our case. Furthermore, it is not fully understood why this syndrome is only seen in girls or young women. May be young gonads are particularly susceptible to stimulation by $\mathrm{TSH}$ or $\mathrm{FSH}^{14}$.

Mousavi et al reported normalization of ovarian appearance in ultrasound 6 months after Thyroxin replacement therapy ${ }^{15}$. Hedayati et al reported regression of cysts with normalization of ovaries after 4 months therapy ${ }^{16}$. In our case there was complete regression of cysts after 3 months and ovaries became normal in size after 4 months and our findings are consistent with others.

Ultrasonography plays an important role in the diagnosis of ovarian hyperstimulation. During ultrasonography careful evaluation is essential to differentiate large cysts with internal multiple septa from large multiple separate thin-walled cysts. Later is the feature of ovarian hyperstimulation giving rise to the formation of multiple thin-walled cysts and the earlier one is the finding of ovarian neoplasm. CT 
scan or MRI also assists in the diagnosis of ovarian hyperstimulation.

Conclusion

Although the syndrome is very rare, hypothyroidism and other endocrine disorders should be considered in the differential diagnosis of adult females presenting with

multicystic ovarian tumors to avoid unnecessary ovarian resection. Young patients with ovarian cysts should be recommended to undergo screening for hypothyroidism.

\section{References:}

1 Montenelli L, Delbaere A, Nappy C et al. A mutation in the follicle-stimulating hormone receptors as a cause of familial spontaneous ovarian hyperstimulation syndrome. J Clin Endocrin and Metab 2004; 89(3):1255-1258

2 Borna S, Nasery A. Spontaneous ovarian hyperstimulation in a pregnant woman with hypothyroidism. Fertil Steril 2007;88(3):701-703

3 Cardoso CG, Graca LM, Dias T. Spontaneous ovarian hyperstimulation and primary hypothyroidism with a naturally conceived pregnancy. Obstet Gynecol 1999;93:809-811

4 Nappi RG, D Naro, Nappi L. Natural pregnancy in hypothyroid woman complicated by spontaneous ovarian hyperstimulation syndrome. Am J Obstet Gynecol 1998;178(3):610-6611

5 Van Wyk J. Grumbach MM. Syndrome of precocious menstruation and galactorrhea in juvenile hypothyroidism: an example of hormonal overlap in pituitary feedback. J Pediatr 1960; $57: 20$

6 Panico A, Lupoli GA, Fonderico F et al. Multiple ovarian cysts in a young girl with severe hypothyroidism. Thyroid 2007;17(12):12891293

7 Gordon CM, Austin DJ, Radovick S et al. Primary hypothyroidism presenting as severe vaginal bleeding in a prepubertal girl. J Pediatr Adolesc Gynecol 1997;10(1):35-38

8 Yamashita Y, Kawamura T, Fuzikawa R et al. Regression of both pituitary and ovarian cysts after administration of thyroid hormone in a case of primary hypothyroidism. Internal Medicine 2001;40:751-755

9 Jhing Shu, Lili Xing, Lingyan Zhang et al. Ignored adult primary hypothyroidism presenting chiefly with persistent ovarian cysts: a need for increased awareness. Reproduc Biol and Endocrine 2011; 9:119

10 Taher BM, Ghariabeh RA, Jarrah NS et al. Spontaneous ovarian hyperstimulation syndrome caused by hypothyroidism in an adult. Eur J Obstet Gynecol Reprod Biol 2004; 112(1): 107-109

11 Hansen KA, Tho SP, Hanly M et al. Massive ovarian enlargement in primary hypothyroidism. Fertil and Steril 1997;67:169-171

12 Merchline M, Riddlesherger MM, Jerald PK et al. The association of juvenile hypothyroidism and cystic ovaries. Radiology 1981;139:77-80

13 Anasti JN, Flack MR. A potential novel mechanism for precocious puberty in juvenile hypothyroidism. J Clin Endocrinol Metab 1998,80(1):776-779

14 Aghajanova L, Lindiberg $M$, Carlsson B. Receptors for thyroid stimulating hormone and thyroid hormones in human ovarian tissue. Reproductive Biomedicine Online 2009;

15 Mousavi AS, Behtash N, Hasanzadeh M et al. Spontaneous ovarian hyperstimulation syndrome caused by hypothyroidism. Cancer Therapy 2005; 3:397- 400

16 Hedayati Emami MH, Molaei Langroudi R, Ghazanfari Amlashi F. Ovarian hyperstimulatyon syndrome and autoimmune primary hypothyroidism in two members of a family. $J$ Clin Case Reports 2012; 2:113 